\title{
SECRETOS, EQUÍVOCOS, NARRADORES Y ESPACIOS: REFLEXIONES SOBRE LA NOVELA CORTA EL ACOSO Y LAS TESIS DE PIGLIA
}

\author{
Ádám András Kürthy \\ Universidad Eötvös Loránd \\ kurthy.adam.a@gmail.com
}

Resumen: El presente artículo toma como punto de partida tres textos de índole teórica de Ricardo Piglia sobre el género del cuento y el de la novela corta, para aplicar sus afirmaciones a la observación de un texto literario, El acoso de Alejo Carpentier, buscando ver así las limitaciones y los aciertos de sus tesis. El acoso, interpretado aquí como novela corta, queda luego brevemente comparado con otras obras carpenterianas, cuento y novela, para ver qué solidez tienen los límites dibujados a base de las ideas de Piglia, con algún que otro suplemento derivado de la contrastación de las tesis con la obra literaria.

Palabras clave: Ricardo Piglia, Alejo Carpentier, El acoso, cuento, novela corta

\section{SECRETS, CONFUSIONS, NARRATORS, AND SPACES: REFLECTIONS ON THE SHORT NOVEL THE CHASE AND ON THE THESES OF RICARDO PIGLIA}

\begin{abstract}
The present article takes three texts of theoretical nature by Ricardo Piglia on the genre of the short story and the novella as its basis, in order to apply his affirmations to the observation of a literary work, The Chase of Alejo Carpentier, aiming to see the limitations and the benefits of Piglia's thesis. The Chase, interpreted here as a novella, is then briefly compared with other Carpenterian works, short stories and a novel, to see how solid the limits are which have been drawn based on Piglia's ideas with the occasional addition ensuing from contrasting the theses with the literary work.
\end{abstract}

Keywords: Ricardo Piglia, Alejo Carpentier, The Chase, short story, novella

DOI: https://doi.org/10.24029/lejana.2020.13.433

Recibido: el 24 de noviembre de 2018

Aceptado: el 1 de marzo de 2019

Publicado: el 19 de febrero de 2020 
A la hora de confrontar un texto teórico sobre los géneros literarios con uno literario, se nos ofrecen dos pautas radicales a seguir. La primera: emplear los preceptos de la teoría al análisis del texto y si este no les corresponde, excluirlo de la clase de obras en cuestión. La segunda: tomar los rasgos del texto analizado como conjunto de atributos de su supuesto género, destacar los puntos en los que la teoría no cumple su deber descriptivo, y, por ende, desecharla como inválida. El propósito del presente trabajo es precisamente evitar estas soluciones categóricas, y observar El acoso de Alejo Carpentier tomando en consideración las ideas de Ricardo Piglia expuestas en varios textos sobre el cuento y la nouvelle, eso es, intentar sacar doble provecho de la lectura asociada: entender mejor la obra de Carpentier y ofrecer apuntes para una visión más comprensiva del género de la novela corta.

Refiriéndome a los textos de Piglia, dudo en emplear la palabra "teoría", porque me parecen más una serie de observaciones muy agudas en relación con las obras que le ocupan, trátese de cuentos o de novelas cortas. En primer lugar, vamos a enumerar algunas ideas suyas que sacan a luz ciertas características fundamentales de la narrativa carpenteriana, para ver luego otros aspectos que se observan en la obra literaria, pero han quedado fuera del alcance de las cogitaciones de Piglia, sin dejar de apuntar a sus aciertos.

\section{Lo que se aplica: secretos y equívocos}

"Un relato visible esconde un relato secreto, narrado de un modo elíptico y fragmentario", dice la tesis fundamental de Piglia (2000: 17). Bien podríamos aplicar el principio a El acoso, modificando un detalle: en su caso, los dos relatos serían el del acosado y el del taquillero, pero ninguno de ellos se cuenta de forma secreta. Sin embargo, dada la manera elíptica y fragmentaria de la narración, son las intersecciones de ambos y la relación causa-efecto las que tienen que ser descifradas en el proceso de lectura. Los principales puntos de enlace son los personajes y las casas de Estrella $^{1}$ y de la vieja: de ellas depende la suerte de ambos protagonistas, y su vida, muerte y acciones ejercen una influencia fundamental sobre el destino de los dos. "A veces, en los relatos, resulta útil hacer el mapa, espacializar la historia cuando uno la lee, porque el secreto debe estar siempre en algún lugar, que puede ser alguien que lo tiene o un lugar donde se lo esconde" (Piglia, 2006: 200) y, de hecho, estos cruces se manifestarían bien en un mapa esquematizado, pero los protagonistas mismos ignoran la interdependencia de su recorrido y de su suerte (el acosado no sabe por qué o por quién le suena conocida la música que escucha en la sala, el taquillero ignora quién es el que entra con retraso y le deja el billete fatídico, etc.). Por tanto, en su nivel, resulta válido el principio siguiente: "lo más importante nunca se cuenta. La historia secreta se construye con lo no dicho, con el sobreentendido y la alusión" (Piglia, 2000: 18). ${ }^{2}$

\footnotetext{
${ }^{1}$ El nombre mismo de Estrella alude a su papel central, a su posición en torno a la cual gravitan los dos hombres. Por otro lado, se puede cotejar el papel de su casa con lo que dice Piglia hablando de Los adioses de Onetti: "El secreto aquí está localizado en un lugar: si pudiéramos entrar en esa casa, saber lo que pasó en esa casa, habríamos comprendido el funcionamiento de la historia" (2006: 200). Piglia se refiere a un ejemplo literario concreto, por tanto, su tesis no se aplica del todo a nuestro caso, sin embargo, una simple modificación del enunciado ayudaría a aplicar lo esencial de su idea a El acoso: si los protagonistas supieran qué había pasado/qué pasaría en esa casa, habrían comprendido/comprenderían... El cambio de la persona verbal será muy importante en lo que sigue.

${ }^{2}$ Nótese el hecho de que tanto los argumentos perfectamente aplicables a la obra de Carpentier como aquellos que precisan modificación provienen de los tres textos de Piglia (además de los ya mencionados véase Piglia,
} 
Y también el otro: "El arte de narrar se funda en la lectura equivocada de los signos" (Piglia, 1999: 111). Pero en este caso son los protagonistas mismos los que leen los signos de manera equívoca: todos los elementos están ahí diseminados entre los puntos comunes (las casas contiguas de la vieja y del taquillero, el velatorio causado por el hambre y el subsiguiente robo del acosado, la música que el acosado oye sin saber, la coincidencia en la predilección hacia Estrella, la ventura del billete con el General con los ojos dormidos), pero ninguno de los dos es capaz de componer el cuadro general, que sería su única posibilidad para entender - y quizá prevenir — lo que les está pasando. Pero este cuadro general no se da con ninguna facilidad, y aun si uno logra recomponerlo, eso sucede solo mediante la narración misma, y no para los personajes, para los que las "pequeñas historias [...] se vinculan $[\ldots]$ de una manera inexplicable" (Piglia 2006: 200).

\section{Lo que se puede ampliar: narrador y espacios}

A propósito de la narración, en este caso, será necesario transfigurar una serie de afirmaciones de Piglia:

A veces el narrador sabe lo que sabe el lector, pero no lo que saben los personajes, a veces un personaje sabe algo que otro no sabe, y sobre esto se construyen las redes y relaciones que conforman las intrigas (2006: 192).

[E]l narrador cuenta una historia que no es la de él, se interesa por una historia que le es ajena [...] (2006: 196).

[E]1 narrador pasa por delante de [...] una escena [...] y trata de entender qué pasa ahí contando apenas con esa percepción parcial a partir de la cual empieza a investigar, a averiguar, trata de entrar en la casa, aunque no siempre lo consigue (2006: 196-197).

[E]1 secreto está entre los que narran la historia, ellos son los que no entienden y se esfuerzan en capturar el sentido. (2006: 202).

En esta serie de citas se percibe una restricción — quizá inadvertida por parte de Piglia - que se da seguramente debido al tipo de textos de los que se ocupaba, y a consecuencia de los cuales pudo haber formulado una teoría de la novela corta onettiana, pero no una de alcance más amplio, dado que implícitamente reduce el círculo posible de los narradores a los intradiegéticos y homodiegéticos (personajes que no son protagonistas de la historia narrada, o a narradores-protagonistas, como atestigua su alusión a Pedro Páramo).

Sin embargo, llega muy cerca de extender su mira en la frase ya citada: "a veces un personaje sabe algo que otro no sabe" (2006: 192), y en su referencia a The Art of Fiction de Henry James, cuya pregunta clave transpone en el texto: "«cuál es el punto de vista en una historia y cuáles son las maneras de acercarse al conocimiento»" (Piglia, 2006: 193). Porque si El acoso resulta inasible para el análisis con las afirmaciones relativas a los conocimientos del narrador, también es cierto que, si desplazamos el énfasis desde la narración a la focalización, a los diferentes niveles de conocimiento entre los personajes del universo

1999), indistintamente de su tema: los hay de sus ideas referentes al género del cuento, así como de lo concerniente al de la novela corta. El hecho no altera su validez, pero plantea la cuestión si Piglia ha logrado dividir del todo las dos categorías, o para ser más justos: si es posible hacerlo en general. 
narrativo $-\mathrm{o}$, visto de otro aspecto, permitimos que el narrador sea exterior al mundo narrado - vamos a ganar un amplio terreno de obras analizables según sus directrices. Porque si parece demasiado banal o intuitivo categorizar la novela corta basándonos exclusivamente en su extensión, no creo tampoco que haya alguien que diga que las novelas cortas solo pueden tener un narrador-testigo o un narrador protagonista.

Por tanto, en El acoso, los secretos no se plantean como algo que el narrador ignora e intenta averiguar, sino como algo que los lectores deben recomponer de los fragmentos turbiamente dispuestos a lo largo del texto, y cuyas interdependencias los personajes mismos ignoran en todo momento. Baste recordar la serie de interpretaciones equívocas y los errores consecuentes del acosado, que deshacen los amparos que podrían salvarlo: se come los manjares de la vieja para no morirse de hambre, con esto, con mantenerse vivo mata a su amparo, tiene que arrojarse a la calle; había delatado a sus compañeros para salvarse la vida (queda expresado que para él la castración equivaldría a la muerte), el resultado es que la casa de la Gestión se ha derrumbado, se le ha quitado la posibilidad de escapar; en la iglesia el cura lo rechaza por el librito de la Cruz de Calatrava, objeto despertador de fe y esperanza de salvación para el acosado, recibido de la vieja negra $-\mathrm{y}$ lo que le ha despertado la fe-, le impide la depuración en la confesión: el libro tiene que ver con la santería, y no tiene cabida en la iglesia. Y, por último, conjetura falsamente: supone que no lo reconocerán por adelgazado, y también que los asesinos lo están esperando fuera, por eso no sale. La salida significaría su salvación, sin embargo, se hace creer que tiene aún varias posibilidades, aunque con este error mantiene en sí otra vez la esperanza para sobrevivir: "Más allá empezará otra época" (258). El acosado se elimina progresivamente los amparos con los actos precedentes hechos para sobrevivir, para salvarse.

Este proceso madura como parte de lo que yo llamo la doble retrospección. En esta, se plasma una inversión mutua del proceso de conocimiento que involucra tanto el proceso ficcional como el proceso de la lectura, ejercitándose al mismo tiempo dentro de la ficción (en un nivel parcial para los protagonistas) y fuera de ella (en el proceso intelectivo de la lectura).

En el caso del acosado: después de ver el cuello con marcas del acné, recuerda aquella ejecución; para el lector: avanzando linealmente en la lectura, se da cuenta de que lo antes leído se entiende a partir de lo expuesto subsiguientemente, que en la cronología es un acontecimiento anterior. Esto puede ocurrir también entre dos partes de la narración extradiegética: vemos antes que ha acontecido algo insólito en la casa de Estrella, por lo cual el taquillero no puede gozarla, y las razones las conocemos después. Así aparece recurrentemente la cita "HOC ERAT IN VOTIS", y también las nociones borrosas del acosado sobre la música, procedentes de haberla oído desde el gramófono del apartamento del taquillero. Pasa lo mismo con el billete falso, con unas complicaciones aún mayores y de importancia crucial. Primero vemos que el protagonista se lo entrega al taquillero y este lo lleva a Estrella quien lo rechaza diciendo que es falso. Después vemos qué dificultades había tenido ella antes por el mismo billete. Al final, en una retrospección hacia ambos acontecimientos, hacia nuestra doble retrospección, por la intervención del policía nos damos cuenta de que el billete es válido. Por lo tanto, todo lo anterior es falso, la razón conductora de varios acontecimientos fatales (el acosado tiene que recorrerlo todo a pie, Estrella tiene que satisfacer al taxista por la falta de dinero) resulta trastornada, y al realizar esta tercera 
retrospección referente al proceso de la lectura, buscando la razón de este desvío fundamental, resulta que no encontramos indicios explícitos: todo parece haber ocurrido al azar.

Por tanto, es preciso transformar otra tesis de Piglia para que sea válida en cuanto a $E l$ acoso: "Lo que quiere decir un relato solo lo entrevemos al final: de pronto, aparece un desvío, un cambio de ritmo, algo externo [...] Entonces conocemos la historia y podemos concluir" (1999: 120). En cambio, en la revelación final de El acoso leemos: “«Uno menos» — dijo el policía recién llamado, empujando el cadáver con el pie. «Además, pasaba billetes falsos» — dijo el taquillero, mostrando el billete del General con los ojos dormidos. «Démelo» — dijo el policía, viendo que era bueno: «Se hará constar en el acta.»" (Carpentier, 1977: $220)^{3}$. Es decir, se nos revela un secreto que ha motivado el desarrollo de los acontecimientos, y se descubre que se ha tratado de un equívoco — posiblemente motivado por el engaño del taxista que quería gozar de Estrella gratis-, pero, a mi modo de ver, esto cambia el sentido solo indirectamente, aunque en un nivel más abstracto su influencia es innegable. Por el contrario, otra afirmación parece escrita aposta para describir este desenlace: "Hay algo en el final que estaba en el origen y el arte de narrar consiste en postergarlo, mantenerlo en secreto y hacerlo ver cuando nadie lo espera" (Piglia, 1999: 122). Este algo podría ser el billete mismo, que aparece al inicio, cuando el acosado entra corriendo y echa el dinero al taquillero: está a la vista de todos, pero su condición verdadera no se revela hasta el último momento.

Es un artificio más que estos acontecimientos logran despertar un auténtico sentimiento de caos mediante una organización textual sumamente compleja y elaborada. Y se podría argumentar que es la voz narrativa la que nos impide ver las conexiones — y será así en último término - , pero dentro de la configuración del texto es la focalización la que rige la dosificación de las informaciones, el narrador se reduce casi a cero: primero, estamos dentro de los pensamientos del taquillero, luego, por gran parte de la obra nos guían las asociaciones del acosado dentro de la Sala de conciertos, donde la música y su percepción guían sus asociaciones a recuerdos del pasado inmediato y más lejano. Quizá el único momento detectable de la voz narrativa independiente de los protagonistas se da en el final citado, porque desde el foco del taquillero sería imposible decir "viendo que [el billete] era bueno" (220). Por la naturaleza de los acontecimientos, y tal vez por la exigencia del final de la historia, aquí se cumple lo que dice Piglia: "la íntima voz que [...] ha marcado el tono y el registro verbal de la historia se identifica y se hace ver" y "se presenta más allá del círculo cerrado de la historia" (1999: 129), eso sí, sin personalizarse en ningún momento. ${ }^{4}$

Piglia menciona el espacio como lugar del secreto central, y, visto de otro ángulo, puede que el espacio sea uno de los aspectos que permitan delinear la novela corta, y trazar sus límites con el cuento y con la novela. Ahora bien, podría argumentarse la obviedad del tema, dado que en una obra de mayor extensión habrá más espacio para describir los detalles del escenario. Pero esto sería mirar el resultado y no el proceso ni el principio de construcción de la obra, porque técnicamente, los elementos van a componer la totalidad, por tanto, las posibilidades descriptivas no provienen de la extensión, sino pueden ser precisamente las descripciones las que originan esta última.

\footnotetext{
${ }^{3}$ Como todas las referencias comprenden la misma edición del texto, de aquí en adelante solo se señalan los número de página correspondientes.

${ }^{4}$ Tampoco se llega a saber si narra en retrospección, o solo sigue a sus protagonistas con el foco — posibilidad bien plausible considerando el esquema temporal de las tres partes-.
} 
Otro contraargumento se nos ofrece a punto si contemplamos la prosa de Carpentier, cuya "voracidad para consumir «lo real» sofoca el discurso a punto de paralizarlo", y que "se pierde en el laberinto de su propio regodeo verbal", resultando en un "desgobierno de la enunciación" (Chiampi, 2001: 99). ${ }^{5}$ No podemos decir que, en género alguno que la obra de Carpentier abarca, se prescinda de las descripciones o del detallismo exuberante. Por ende, no se nos deja establecer un esquema tripartito simplista: 1) cuento = muy poca descripción; 2) novela corta $=$ poca descripción; 3) novela $=$ mucha descripción. $\mathrm{Y}$ entonces, podría resultar fructuoso examinar un espacio ejemplar en El acoso yuxtaponiéndolo a uno semejante que aparece en la novela de Los pasos perdidos para ver la posible presencia —o ausencia— de rasgos distintivos.

Para ello, vamos a elegir un espacio que las dos obras comparten. Generalmente, en la configuración de las escenas en que se escucha música (trátese de concierto público, privado, de función teatral o de retransmisión radiofónica) o se ve una representación, se puede observar que esto funciona como incentivo para las reflexiones, y los motivos musicales rigen los pensamientos, las intro- y retrospecciones, por tanto, el espacio primario sirve como trasfondo para la música, que a su vez contribuye a abrir camino a la aparición de espacios secundarios (de nuevo, mentales, intro- o retrospectivos). Por consiguiente, me parece conveniente observar los momentos y las zonas límite, así como la configuración de estos espacios primarios en los instantes anteriores a que el espacio deje paso a la música y a la introducción de espacios secundarios.

En El acoso, tenemos un espacio liminar muy marcado, puesto que el taquillero está en el foco, y con él, la taquilla, lugar de pasaje por excelencia hacia el interior de la sala, dado que no logra incluirse por completo en el mundo de adentro, pero, a la vez, queda desconectado del mundo de afuera, e incluso es un espacio restringido dentro del marco transitorio de la antesala. Y estas limitaciones se hacen patentes sin demora. Partimos del libro, objeto no nombrado, presente en su texto, en el proceso de la lectura, y luego interviene el portazo (señal de límite), estableciéndose así una dinámica ensimismamiento-sobresalto. El mundo de afuera también se entrevé (el público refrescándose en el entreacto) o incluso se entrehuele: "el olor a mojado, a verde de álamos, a gramas regadas [...] mezclándose con alientos de tierra y de cortezas" (125). Queda evidente la posición marginal del taquillero correspondiente a la posición de su microespacio-, a la par de su falta de autoestima que se corrobora en las acciones de lo exterior, que pasa por alto sobre él: el público (la mujer ajustándose el vestido), los acomodadores (grupo con el que podría tener más contacto siendo empleados de la sala), e incluso sus compañeros más cercanos: "aquel taquillero distinto a todos los demás taquilleros" (126). Este aislamiento tanto psicológico como espacial se afilia a una dualidad emocional: 1) el "pueril orgullo de haber entendido aquel texto" (125) y un poco más tarde, "Si estaba ahí [...] era por alcanzar el entendimiento de lo grande [...] Esa conciencia le devolvía su orgullo frente a las espaldas muelles" (127); 2) la humillación, comprobada en su "inexistencia para las mujeres" (126), resultante en su reticencia: "La mirada huyó lo cercano inalcanzable" (126) o incluso en una voluntad de venganza: "Quiso humillar a la del zorro" (126).

\footnotetext{
${ }^{5}$ Para ser exactos, los calificativos de Chiampi se refieren al narrador de la parodia que Cabrera Infante hace de Carpentier, no obstante, las tendencias del narrador carpenteriano quedan bastante bien señaladas en esta exageración.
} 
Es justamente la mirada en huida la que se desvía hacia afuera, hacia la casona del Mirador, y esto propicia la ocasión para que empiece a tejerse la red espacial de la acción en este mismo momento se produce el segundo momento de sobresalto, el primer truenoAparece el espacio de la vieja y del refugio del acosado; también es curioso observar que este desplazamiento de la mirada trae consigo un desplazamiento espacio-temporal, al pueblo y a los recuerdos del taquillero.

El protagonismo del espacio primario queda restringido en el tiempo por las exigencias de la acción: el fin del entreacto lleva a la gente a entrar y a ocupar sus butacas en este momento se divisa también el espacio interior, el de la sala, hasta "el primer gesto del director" (128) —. La visión se justifica con la puerta entornada "para que los morosos pudieran entrar en puntillas" (128). En este instante llega el tercer sobresalto, el del "frenazo brutal" (128). Se pasa a un movimiento vertiginoso, acentuado por la restricción del campo visual por las rejas: se escucha una "voz presurosa", y se ven los dedos que arrojan el billete "por entre los barrotes de la taquilla" (128); por un segundo, el foco parece desplazarse, siguiendo la mirada del recién llegado: "Viendo que los talonarios estaban guardados y que se buscaban llaves para sacarlos, el hombre se hundió en la obscuridad del teatro, sin esperar más" (128). Es él ahora "el hombre", y el otro elemento (el taquillero) se reduce a un mero pronombre ("se buscaban"). En esta misma perspectiva pasan los dos perseguidores del acosado, y, un poco más tarde, al taquillero se le denomina como "el de las rejas" (129). Sin embargo, el billete reclama el centro de atención: el taquillero hace inferencias a base de lo visto, luego su imaginación se pone en marcha, con un espacio ilusorio muy peculiar: "Un puente apartaba las rejas, atravesaba las paredes, se alargaba hacia la que esperaba [...] en la penumbra de su comedor" (129). Así que, por último, se deja constancia también de la existencia de Estrella, y la imagen — junto al poder del billete - lleva a la interrupción de la conexión del taquillero con la sala, orientándolo hacia afuera: "Un gesto [...] apartó la cortina de damasco que lo separaba de la sala, donde el silencio había inmovilizado a los músicos en posición de ataque" (129-130). A los primeros compases, "el libro quedó cerrado de un manotazo" (130), con el último sobresalto del capítulo, se podría decir. Vuelven los olores, como otra guía hacia afuera, y el desplazamiento conlleva de nuevo un cambio temporal al plano espacio-temporal de los recuerdos. El trasfondo emocional (su orgullo y su devoción hacia la música) le sirve como excusa para la escapada hacia "la casa sin relojes"6 (131).

Por tanto, se ve que en la primera media docena de páginas se presentan todos los elementos posteriores $-\mathrm{o}$ anteriores, en la cronología de los acontecimientos-: el acosado, la vieja y su casa, Estrella y su casa, y los acosadores. Todos están condensados al mismo espacio, como mínimo, mediante la asociación.

Ahora bien, la escena que más automáticamente podría ofrecerse para la comparación en Los pasos perdidos es la del narrador-protagonista que, huyendo de la lluvia, entra en la sala de conciertos, no del todo fortuitamente, encaminado por sus recuerdos. Se podría trazar la analogía entre su entrada y la del acosado: ambos tienen conocimiento previo del lugar, y ambos son guiados por cierta urgencia - aunque de gravedad bien distinta- Sin embargo, hay varias diferencias por las que me parece mejor elegir otro momento de la novela. Si bien

\footnotetext{
${ }^{6}$ Nótese la inmediata asociación de la connotación temporal al nombrar el espacio de destino del taquillero saliendo.
} 
la diferencia de la categoría del narrador (extra- y heterodiegético, restringido a la percepción focal en $\mathrm{El}$ acoso, frente al narrador-protagonista que filtra todo a través de sus propósitos y puntos de vista) persiste a lo largo de ambos textos: aquí no tenemos un microespacio apartado, intermedio dentro de lo intermedio, ni estamos en una posición textual inicial, y eso deniega al fragmento de la novela un privilegio del que su intuido equivalente apenas analizado sí dispone. En consecuencia, vamos a ver otro homólogo suyo, el espacio del íncipit de Los pasos perdidos.

Aquí, el espacio aparece en seguida en clave del tiempo, tal como se subraya en el inicio y el final de la primera frase, circundando la nomenclatura de la descripción espacial: "Hacía cuatro años y siete meses [...] tenía la casi penosa sensación de que el tiempo se hubiera revertido" (Carpentier 1994: 9) ${ }^{7}$. A partir de este punto, se establece una duplicidad: la mayoría de la descripción la ocupan los elementos de cierto paisaje (el farol, el rosal, la jaula vacía, los olmos, el río, la magnolia enana, el banco de piedra), pero con incisiones que aluden a que el escenario no es lo que es. A propósito del banco de piedra, el narrador dice: "que hice sonar a madera de un taconazo", hay una sospechosa "cortina de color vino", se refiere al olmo y los tiempos de su plantación, "cuando todos colaborábamos en la obra común" (9). Se detecta aquí cierta escisión de una unidad. La duplicidad también aparece en los elementos humanos de la descripción: los esclavos marcados, las amazonas y los soldados heridos están en las "sombras hediondas a mastic, a fieltros viejos, a sudor resudado en las mismas levitas" (9), palpándose una repetición, tan crucial más tarde. La situación se aclara solo en este punto: "A tiempo salí de la luz [...] un pájaro cayó en la escena” (9). Se aclara, pues, que se trata de una sala de teatro, cuyas partes principales son el palco y el escenario. Pero la posición del narrador-protagonista queda fuera: no es ni el uno, ni el otro, es una situación marginalizada, aunque con vistas a las partes principales. Los que están detrás de los bastidores (y el narrador sí está) no forman parte de la función teatral. En este momento lo roza "el miriñaque de [su] esposa", por tanto, se establece una relación familiar a través del contacto en el espacio: "me hallaba [...] donde le tocaba entrar, estrechándole el ya angosto paso" (9). El contacto espacial propicia la salida del narrador-protagonista: "Por molestar menos me fui a su camerino, y allá el tiempo volvió a coincidir con la fecha, pues las cosas bien pregonaban que cuatro años y siete meses no transcurrían sin romper, deslucir y marchitar" (9). Por tanto, se llega a otro espacio primario, si lo consideramos estrictamente, y a la vez aparece el primer elemento repetitivo (la referencia al lapso temporal). Por otro lado, el camerino también podría compararse a la posición ni dentro-ni fuera de la taquilla, especialmente dado que precede la asociación del teatro a la "prisión de tablas de artificio" para Ruth, y a los "árboles de mentira" (10).

Si ampliamos un poco la mira, y concedemos un espacio textual más amplio al íncipit, podemos encontrar huellas del mismo ideario: "Así, para Ruth, lejos de ser una puerta abierta sobre el vasto mundo del Drama - un medio de evasión- este teatro era la isla del Diablo" (10) (sigue la retrospección al curso de los 4 años y 7 meses, pero el espacio no cambia, como máximo hacia el cementerio, en el imaginario de la aniquilación, también resaltada en el texto). Se habla de "breves fugas", de "escaso alivio" (10) y del "automatismo del trabajo

\footnotetext{
${ }^{7}$ Como todas las referencias comprenden la misma edición del texto, de aquí en adelante solo se señalan los números de página correspondientes.
} 
impuesto" (11). El íncipit se cierra sin lugar a dudas con las palabras finales de esta reflexión: "Sonaba el disparo, caía el falso pájaro del segundo tercio de bambalinas, y se daba por terminada la Convivencia del Séptimo Día", visto especialmente desde la frase siguiente: "Hoy, sin embargo, se había alterado la regla dominical" (12), a poca distancia de la primera mención de Mouche.

Bien mirado, pues, vemos que el íncipit, o la presentación del espacio inicial, es incluso más corto en Los pasos perdidos que en El acoso — dependiendo del criterio con el que lo delimitamos-, y vemos algunos puntos comunes, ya que en Los pasos perdidos también se exponen ciertos elementos fundamentales a lo largo de toda la acción: el afán de evasión, la estrechez del matrimonio y la monotonía, encapsuladas en el espacio teatral, el "angosto paso" (9), en una conexión literal con el paso de la selva, y, por último, el escenario, un espacio que es lo que es y no lo es a la vez, que es real en el pacto ficcional del teatro, pero montado y construido fuera de él. Este último aspecto podría aplicarse también a los espacios del viaje: son escenarios que sirven como trasfondo para montar los preconceptos del narrador-protagonista que se nos van exponiendo disfrazados como experiencias nuevas.

Al contrario, vemos también que muchos elementos quedan fuera del alcance de la novela: si lo forzamos un poco, podemos decir que aparece Mouche, pero seguramente faltan piezas clave: Rosario, el minero griego y el Adelantado, tanto como la idea misma del viaje. Esto se debe a la numerosidad de los elementos posteriores o a su proporción distinta en las dos obras. La diferencia también queda reflejada si contemplamos la posición de los espacios analizados en la escala respectiva de los espacios.

En El acoso, tenemos los espacios primarios, que serían los que tienen papel en los acontecimientos durante el curso del concierto (por tanto, los "más importantes"). Estos se ubican en la ciudad de La Habana (Wyers Weber, 1963: 440), eso es, podría encontrarse su identificación en la realidad extraficcional - si es que importa nombrar su localidad si en la obra no se identifican como partes de tal- Los secundarios aparecen exclusivamente en las retrospecciones, tanto las del taquillero sobre su niñez como las del acosado sobre sus acciones en los tiempos del Tribunal, y a veces (pero no en todo caso) quedan situados fuera de la capital: el lugar de procedencia del taquillero evocado en sus memorias, y el del acosado, Sancti-Spiritus. ${ }^{8}$ Bien mirado, hay solo tres espacios primarios. 1) la Sala de conciertos - aunque esta podría dividirse a su vez en varias unidades menores: en la entrada y la taquilla (puesto del taquillero), y adentro, en la sala propiamente dicha (lugar de la ejecución de la Sinfonía y del protagonista) —; 2) la casa de Estrella (en la vana visita del taquillero); 3) el recorrido del taquillero (desde dicha casa, tocando el mercado, hasta la vuelta a la Sala). En el campo visual de este último se incluye la casa de la vieja, por quien el taquillero siente preocupación: "Necesitaba saberla viva, en la noche, por rito de purificación" (Carpentier, 1977: 145).

El apartado número II de la novela comienza con este mismo escenario, adentrándose en la habitación de la vieja: la contigüidad textual encubre diestramente el hecho de que aquí ya se trata de memorias - y por ello, de espacio secundario-: en el foco está el acosado, refugiado en la Sala, que rememora sus propios antecedentes que lo llevaron hasta allí. Y todo

\footnotetext{
${ }^{8}$ También una ciudad existente; con nombrarla, se le da una carga simbólica de este lugar, tal como lo subrayan las palabras de Skrapits (2008: 260).
} 
lo que viene después, hasta el momento final del concierto y la huida deseada pero truncada, se sitúa en el plano secundario, en la mente del acosado: el espacio primario de la Sala aparece de manera intermitente como respaldo y base de sus congojas y asociaciones, y finalmente como escenario de la ejecución.

Tomando en cuenta la dimensión, podemos hablar de microespacios, los espacios de escenas concretas, en la mayoría interiores. Los más destacados de El acoso serían: el edificio alto (lugar de residencia del taquillero), la casa de la vieja (refugio del acosado), la Sala de conciertos (espacio de los momentos finales narrados en las partes I y III, con la posible subdivisión ya mencionada); la casa de Estrella (punto de referencia para el acosado y el taquillero), la Casa de la Gestión y la Iglesia (paradas del acosado en la espera de la salvación), la playa y el café próximo a la Sala de conciertos (inicio del acoso final).

En los niveles más amplios, conviene observar que el lugar del contacto entre taquillero y acosado se reduce a un triángulo, más la casa de Estrella. El triángulo comprende el edificio alto en que vive el taquillero, la casa de la vieja, en donde se refugia el acosado, y la Sala de conciertos. Desde la Sala y desde el edificio (lugares fijos del taquillero) se ve la casa de la vieja (el hombre se preocupa por ella al ver las velas), en donde el acosado oye la música que sale del apartamento del taquillero. Por tanto, existe la comunicación dentro del triángulo, pero siempre en canales restringidos, de manera parcial, sin la totalidad de la percepción. Los puntos comunes, en que ambos están presentes en algún momento, son la casa de Estrella (en sucesión) y la Sala (contemporáneamente).

En total, la narración cuenta con una decena de escenarios, o una docena si agregamos las memorias de la ejecución del primer delator y la prisión del acosado.

Por el otro extremo, Los pasos perdidos contiene como mínimo tres áreas mayores marcadamente diferentes: la ciudad de donde sale el protagonista, que "no necesita de mayor ubicación" (Carpentier, 1994: 261), presumiblemente norteamericana; luego, la capital latinoamericana y las ciudades provincianas; y el espacio del viaje en autobús (Los Altos, Valle de las Llamas) y más tarde en barco, en la selva (Puerto Anunciación) con el río y con el paso hacia Santa Mónica de los Venados. Detallando las subdivisiones de estos macroespacios, ya nos falta poco para llegar a la cantidad de una docena, y, a diferencia de lo anterior, aquí cada espacio de nivel intermedio dispone de ulteriores ramificaciones porque, dentro de los espacios, las escenas se concretizan en escenarios de un nivel inferior. Acabamos de ver la escena inicial del teatro, y luego sigue la casa del narrador-protagonista, la casa de Mouche, la sala de conciertos o el museo en la primera ciudad, el hotel con sus diferentes partes y la tienda con la calle de la capital latinoamericana, el mismo autobús o el barco de los viajes, y con eso, sin una enumeración completa, ya se puede apreciar el fondo.

Parece, pues, contundente el desmarque de la novela frente a la novela corta en la densidad / proporción de los elementos implantados en cada escenario menor que se conectan con otras partes del texto, y también en cuanto a la ramificación y la multiplicación de los niveles según la dimensión de los espacios. Por otro lado, si dejamos de observar la diferencia novela-novela corta y volvemos la atención hacia la relación novela corta-cuento, hemos visto que, a la hora de analizar El acoso de Alejo Carpentier, varias afirmaciones de Piglia se han mostrado afines a la obra tanto entre sus tesis referentes al cuento como a la nouvelle, y los criterios discutidos en la primera parte de este trabajo también muestran que la diferenciación es muy delicada. La cuestión está lejos de tener una respuesta definitiva, pero, tal vez, 
llamando la atención a la importancia de la focalización frente a la mera mención del narrador, y escrutando los diferentes niveles de la espacialidad, las delimitaciones podrán volverse algo menos borrosas. Vamos remendando y completando las ideas, en la espera de acercarnos más y más a una definición certera. $\mathrm{O}$, como menos, la refutación de las propuestas aquí expuestas restringirá el círculo posible de los acercamientos relevantes para la diferenciación.

\section{Bibliografía}

CARPENTIER, Alejo (1977): “El acoso". En su libro Dos novelas. La Habana, Letras Cubanas: 123-220.

--- (1994): Los pasos perdidos. Buenos Aires, Editorial Quetzal.

CHIAMPI, Irlemar (2001): “Barroquismo y afasia en Alejo Carpentier". En su libro Barroco y modernidad. México, Fondo de Cultura Económica: 98-117.

PIGLIA, Ricardo (1999): "Nuevas tesis sobre el cuento". En su libro Formas breves. Buenos Aires, Temas Grupo Editorial: 103-134.

--- (2000): “Tesis sobre el cuento". Guaraguao. Revista de Cultura Latinoamericana IV/11: 17-19.

--- (2006): "Secreto y narración. Tesis sobre la nouvelle". En Eduardo Becerra, El arquero inmóvil. Nuevas poéticas sobre el cuento. Madrid, Páginas de Espuma: 187-205.

SKRAPITS, Melinda (2008): "Sentido y sensibilidad. La música traducida a palabras. El discurso musical en El acoso de Alejo Carpentier". En Amelia Blas, Gabriella Menczel y László Scholz. (eds.), El reverso del tapiz. La traducción literaria en el ámbito hispánico. Budapest, Instituto Cervantes: 257-265.

WYERS WEBER, Frances (1963): “El Acoso: Alejo Carpentier's War on Time”. PMLA (New York) (78) 4. (Sep. 1963): 440-448. Recuperado de: https://www.jstor.org/stable/461257 [26/01/2020]. DOI: https://doi.org/10.2307/461257

(C) Ádám András Kürthy

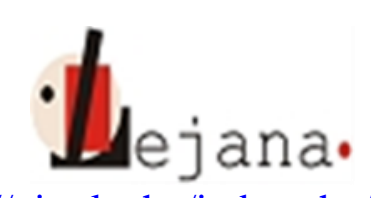

http://ojs.elte.hu/index.php/lejana

Universidad Eötvös Loránd, Departamento de Español, 1088 Budapest, Múzeum krt. 4/C 